\title{
Diffusion with Variable Production Lead Times*
}

\author{
Chung-Yi Tse ${ }^{\dagger}$
}

July 16, 2007

\begin{abstract}
When there is not one obvious candidate technology, entrants to a new industry face a non-trivial choice between longer lead times in the setting up of production and a better chance that the technology could successfully deliver. This paper shows how this tradeoff may yield gradual diffusion. Diffusion is more protracted in industries where learning opportunities are more bountiful. The equilibrium minimizes the long-run equilibrium price, just as in the standard Marshallian model of a competitive industry. The market structure does not seem to affect the rate of diffusion with the monopoly choosing the same rate of diffusion that prevails in competition despite restricting output.

JEL classificaions : L10, L11, O33.
\end{abstract}

Keywords : Technology choice, Production lead times, Diffusion, Learning by doing.

*This paper grew out of discussions with Boyan Jovanovic. His comments and suggestions were pivotal to the completion of the paper. I have also benefited from the helpful comments and suggestions by an anomyous referee. All remaining errors are mine.

${ }^{\dagger}$ Correspondence : School of Economics and Finance, University of Hong Kong, Pokfulam Road, Hong Kong; email : tsechung@econ.hku.hk; phone : (+852) 2859-1035; fax : (+852) 2548-1152. 


\section{INTRODUCTION}

Often entrants to a new industry face a substantial amount of uncertainty as to what technology may work in the new setting. The natural response is to adapt and try out technologies that have proven to work elsewhere. But there is usually not a single obvious candidate technology. There are technologies that call for few adaptations and short lead times before production may be attempted. There are technologies that require substantially more adaptations and longer lead times. But more adaptations and longer lead times could improve the chance that the chosen technology would ever work in the new setting at all. These ideas go back to Alchian (1959), who argued that the payoff for firms to choose a longer lead time is that production costs tend to be lower to follow.

How long to wait out before production should be attempted is an important and conscious decision for entrants. In this paper, we study how these decisions of nonatomistic producers collectively yield gradual diffusion. The period of time to wait out before attempting production is a period of learning by doing. Our analysis assumes that it cannot be substituted at all by heavier investment in physical capital or in personnel. This is probably somewhat extreme. But empirical evidence abound to support the notion that productivity increases with experiences (Bahk and Gort (1993), among many others). This suggests, at the very least, that firms cannot entirely substitute away time as an input in the setting up of production.

The investment in terms of foregone income for technologies with shorter waiting times is lower. But these technologies could be less likely to work at all. That the benefits and costs of technologies with various waiting times could just cancel out in equilibrium is a distinct possibility. In this case, there are staggered entries into full-fledge production and gradual diffusion in equilibrium. We show that under some appropriate parameterization of the demand curve, output follows the familiar 
S-shaped diffusion curve, and the density of technology choice is bell-shaped.

Clearly, the option to wait out longer before attempting production should only be taken up if it yields a large enough improvement in the chance that production could be successfully commenced. The analysis to follow shows that in competitive markets, this turns into the condition that the last technology to be adopted is associated with the lowest long-run equilibrium price - a result only to be expected. What is somewhat unexpected is that a monopoly would choose the same range of technologies to try out as well. True, the monopoly restricts output. But the output restriction is entirely through running each technology less intensively, while the range of technologies to be tried out coincides with the range of technologies selected in competitive markets.

This paper is, by all means, not the first paper to invoke learning by doing to help generate gradual diffusion. The defining difference between the present paper and previous analyzes that include, among others, Jovanovic and Lach (1989) and Reinganum (1981), is that we assume that the benefits of learning are entirely internal, whereas previous analyses concentrate on the spillovers of benefits from early to late entrants. There are two major differences in the implications. First, where the benefits of learning by doing are external, market size and the distribution of consumers' willingness to pay could play decisive roles in the pace of diffusion. In a larger market and in a market with more high income consumers, more firms would enter initially. If the experiences acquired by early entrants spillover to subsequent entrants, the rate of entry increases even further, speeding up the diffusion to low income consumers as a result (Jovanovic and Lach (1989); Matsuyama (2002)). In the present model, where the benefits of learning are purely internal, the pace of diffusion is a function of technology only, but not of demand. And how long it takes for the product of the new industry to diffuse to any given consumer does not depend on market size nor the distribution of income. Second, entry and diffusion tend to be more gradual in 
models of external learning when productivity increases rapidly with industry-wide experience. The reason is that if entries instead cluster temporally, a deviating firm that chooses to wait just a bit longer before entry would become an order of magnitude more productive (Jovanovic and Lach (1989). We show that the opposite is true in this paper. That is, with internal learning, entry tends to be concentrated temporally in case the benefits of learning increase rapidly with experience and flattens out quickly.

As a theory of how different technologies would be simultaneously adopted, the paper is related to the literature on appropriate technology (Basu and Weil (1998)). This paper is closest to Jovanovic (2004) who also considers how differences in production lead times among firms result in staggered entries and exits and gradual diffusion. The major difference is that the present paper models lead times as conscious decisions of firms, whereas Jovanovic (2004) assumes that production lead times for individual producers follow the same stochastic process.

The rest of the paper is organized as follows. The next section explains the basic setup and presents a 2-technology example. Section 3 generalizes to analyzing where there is a continuum of technology. Section 4 illustrates, via two examples, how the lifecycle of the industry depends on whether learning is primarily active in the sense of Ericson and Pakes $(1995,1998)$ or passive in the sense of Jovanovic (1982). Section 5 explains how in the present model the roles played by demand on the rate of diffusion is decidedly minor. Section 6 compares the monopoly's technology choice with a competitive industry's technology choice. Section 7 concludes.

\section{TECHNOLOGY CHOICE}

We study an industry that faces a demand curve $q=D(p)$, where $q$ is industry output and $p$ is industry price. Potential entrants into the industry suddenly become aware of its existence at date zero. There is a menu of technologies indexed by $z-$ 
the waiting time until production starts. If it commits to technology $z$, the firm's output depends on the firm's age, $t$, as follows:

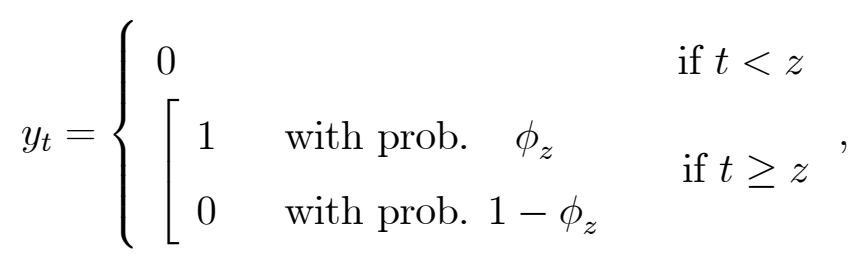

where $\phi_{z}<1$ is the probability that technology $z$ would succeed for the given firm. The outcomes are independent over firms, so that with a continuum of firms, $\phi_{z}$ is also the fraction of such firms that succeed to produce. The firm must be present in the industry throughout the period to learn and to adapt the chosen technology for production in the industry. While it develops its technology the firm forgoes income of $w$ per unit of time.

We shall assume that immediate production is infeasible for any of the technologies on the menu, so that $\phi_{0}=0$. At some point $L, \phi_{z}$ starts to rise above zero and asymptotes to a value not exceeding unity. Figure 1 plots two possible $\phi_{z}$.

We shall show that no successful producers will choose to exit. This means that output can only rise or, at least remains constant. Thus $p_{t}$ must fall over time. This implies that all entries take place at $t=0$, and firms whose technologies fail to deliver at the chosen $z$ would exit the industry permanently to return to earning $w$ elsewhere. The present value, $V_{z}$, that goes with technology $z$ is

$$
V_{z}=e^{-r z}\left(\left[1-\phi_{z}\right] \frac{w}{r}+\phi_{z} \int_{z}^{\infty} e^{-r(s-z)} p_{s} d s\right)
$$

where $r$ is the rate of interest. Let $N_{z}$ be the mass of firms adopting technology with a waiting time not longer than $z$. If $N$ is continuous, we define $n_{z}=N_{z}^{\prime}$. Industry output at $t$ is

$$
q_{t}=\int_{0}^{t} \phi_{z} d N_{z}
$$

Equilibrium.- -is the pair of functions $(p, N)$ that satisfies: 


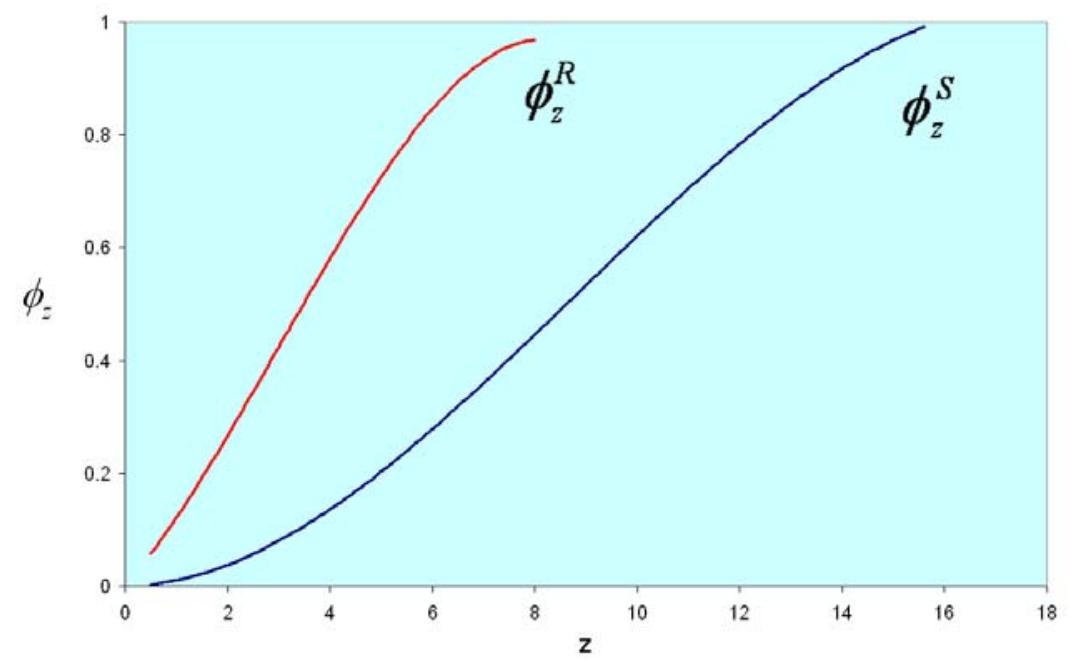

FIG. 1. Two learning curves

1. Market clearing:

$$
q_{t}=D\left(p_{t}\right)
$$

2. Free entry:

$$
V_{z}=\frac{w}{r}
$$

for all $z$ in the support of $N$ and

$$
V_{z} \leq \frac{w}{r}
$$

outside the support of $N$.

Note from (4) and (2) that the successful producers are strictly better off if they remain in the industry. Hence indeed no successful entrants would choose to exit.

\subsection{One-technology example}

As a preamble to the analysis for which firms face genuine tradeoffs in their technology choices, it is instructive to examine the simpler case in which no such tradeoffs 
exist. Suppose

$$
\phi_{z}= \begin{cases}0 & \text { if } z<\widehat{z} \\ \widehat{\phi} & \text { if } z \geq \widehat{z} .\end{cases}
$$

Choosing a $z<\widehat{z}$ is a lost cause and a $z>\widehat{z}$ is wasteful. Technology choice is trivially at $z=\widehat{z}$ for all entrants. The zero-profit condition is

$$
\frac{w}{r}=e^{-\widehat{z}}\left([1-\widehat{\phi}] \frac{w}{r}+\widehat{\phi} \frac{p}{r}\right)
$$

where $p$ is the equilibrium price at time $\widehat{z}$ and thereafter. Rearranging,

$$
p=\left(1+\frac{e^{\widehat{r}}-1}{\widehat{\phi}}\right) w .
$$

There is a percentage markup of price over marginal cost equal to $\frac{e^{\widehat{r z}}-1}{\widehat{\phi}}$, which is increasing in $\widehat{z}$ and decreasing in $\widehat{\phi}$.

All $N_{\widehat{z}}$ entrants at time 0 would stay until $t=\widehat{z}$. Then at $t=\widehat{z}$, a fraction $\widehat{\phi}$ of the entrants begin production, and so output from thereafter is $q_{t}=N_{\widehat{z}} \widehat{\phi}$. Since firm size is fixed at 1, equilibrium price is a function of technology only. The demand curve determines only the number of entrants, $N_{\widehat{z}}$, via the condition:

$$
N_{\widehat{z}}=\frac{1}{\widehat{\phi}} D\left(\left(1+\frac{e^{r \widehat{z}}-1}{\widehat{\phi}}\right) w\right) .
$$

Because technology choice is trivially at $\widehat{z}$ for all entrants, there would only be trivial dynamics in $p_{t}$ and $q_{t}: p_{t}=D^{-1}(0)$ and $q_{t}=0$ until $t=z$; thereafter $p_{t}$ falls to and stays at the level given in (5), while $q_{t}$ rises to and stays at $N_{\widehat{z}} \widehat{\phi}$. Further all exits take place at the same time $z$. True, firms do experience different fortunes over time as only a fraction of all entrants successfully make the transition to become bona fide producers. But such is not enough to paint a picture where $p$ falls and $q$ rises gradually, and technology choices are dispersed and exits are staggered. 


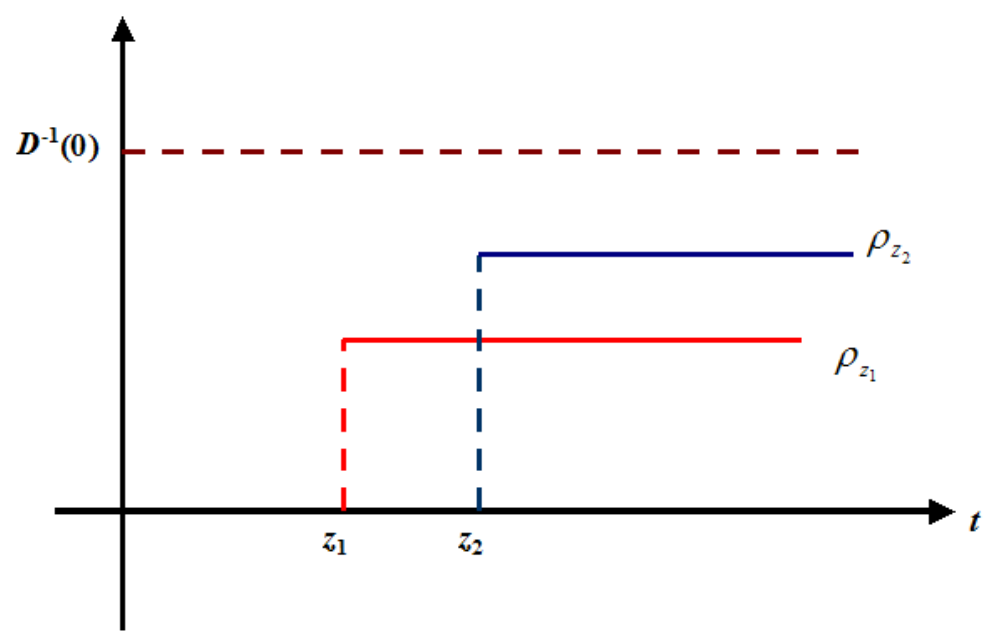

FIG. 2. Identical technology choice

\subsection{Two-technology example}

Since everyone prefers a shorter waiting time and a higher success probability, we can rule out choices of $z$ at which $\phi_{z}$ is non-increasing. But if $\phi_{z}$ is strictly increasing over at least a given range of waiting times, firms would face genuine tradeoffs between shorter waiting times and higher success probabilities. To analyze this tradeoff in the simplest setting possible, assume

$$
\phi_{z}= \begin{cases}0 & \text { if } z<z_{1} \\ \phi_{1} & \text { if } z_{1} \leq z<z_{2} \\ \phi_{2} & \text { if } z \geq z_{2}\end{cases}
$$

for some $z_{2}>z_{1}$ and $\phi_{2}>\phi_{1}$. Each firm's technology choice is between the more ambitious $z_{1}$ and the more certain $z_{2}$.

By (5), if all entrants adopt the same technology $z_{i}, i=1$ or 2 , the equilibrium price from time $z_{i}$ and thereafter would be

$$
\rho_{z_{i}} \equiv w\left(1+\frac{e^{r z_{i}}-1}{\phi_{i}}\right) .
$$


First, assume $\rho_{z_{2}}>\rho_{z_{1}}$, as depicted in figure 2. Say all entrants adopt technology $z_{2}$. But then there must be positive expected profit for a deviating firm that adopts technology $z_{1}$. This firm only requires $p_{t}=\rho_{z_{1}}$, for $t \geq z_{1}$, to break even. But in an "all $z_{2}$ equilibrium",

$$
p_{t}= \begin{cases}D^{-1}(0) & t<z_{2} \\ \rho_{z_{2}} & t \geq z_{2}\end{cases}
$$

Moreover, there cannot be an equilibrium in which both $z_{1}$ and $z_{2}$ are adopted. In any such equilibrium, for $t \geq z_{2}, p_{t}=\rho_{z_{2}}$ must still hold. In case both $z_{1}$ and $z_{2}$ are chosen, we must have $q_{z_{1}}<q_{z_{2}}$; thus for $t \in\left[z_{1}, z_{2}\right), p_{t}>\rho_{z_{2}}\left(>\rho_{z_{1}}\right)$. Indeed, in case $\rho_{z_{2}}>\rho_{z_{1}}$, the unique equilibrium is where all entrants adopt $z_{1}$. This is equilibrium since any deviating firm adopting $z_{2}$ requires $p_{t}=\rho_{z_{2}}$ for $t \geq z_{2}$ to break even, but for all such times, $p_{t}$ remains equal to $\rho_{z_{1}}\left(<\rho_{z_{2}}\right)$ in equilibrium.

Next suppose $\rho_{z_{1}}>\rho_{z_{2}}$ instead, as depicted in figure 3. Can equilibrium still be all entrants choosing the same shorter waiting time $z_{1}$ ? A deviating firm choosing $z_{2}$ in an "all $z_{1}$ equilibrium" can more than break even since in such an equilibrium, $p_{t}=\rho_{z_{1}}>\rho_{z_{2}}$, for all $t \geq z_{1}>z_{2}$. The equilibrium cannot be all entrants choosing the same longer waiting time $z_{2}$ either if the maximum price the market will bear, $D^{-1}(0)$, is sufficiently high. In an "all $z_{2}$ equilibrium", a deviating firm adopting $z_{1}$ could sell at $p_{t}=D^{-1}(0)$, for $t \in\left[z_{1}, z_{2}\right)$. If this $D^{-1}(0)$ is sufficiently high, the expected discounted profit could well rise above $w / r$ for the deviating firm. In this case, both $z_{1}$ and $z_{2}$ be would adopted in the unique equilibrium.

In this 2-technology example, the nature of equilibrium depends crucially on whether or not $\rho_{z_{2}}<\rho_{z_{1}}$ holds, or equivalently,

$$
\frac{\phi_{2}}{\phi_{1}}>\frac{e^{r z_{2}}-1}{e^{r z_{1}}-1} .
$$

If the condition is met, both technologies would be adopted in equilibrium. Otherwise, the option to wait out a longer period of time before attempting production would 


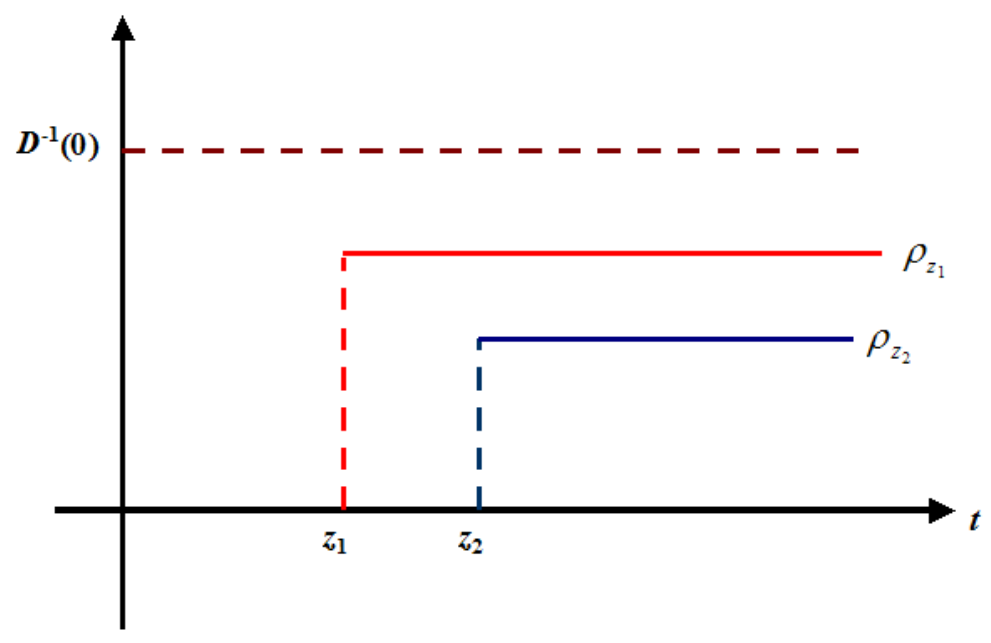

FIG. 3. Dispersed technology choice

not be taken up by firms. Condition (6) is only met if $\phi_{2}$ exceeds $\phi_{1}$ by more than an amount that is a function of the forgone income of waiting out longer. In this case and only in this case, firms find it worthwhile to wait out longer than is absolutely necessary before attempting production.

Moreover, in the 2-technology example, the last technology adopted is the one with the lower $\rho_{z}$. Intuitively, entry should not have stopped if the long-run equilibrium price can possibly be lower still. But it should not have proceeded past where the long-run equilibrium price is lowest either.

Finally, in this example, as in where the technology choice is trivially at a given $z$, demand plays no role in determining $\left\{p_{t}\right\}$ as long as there is a sufficiently high $D^{-1}(0)$. Would these conjectures apply in general where $\phi_{z}$ is continuously increasing over a range of waiting times? To this we now turn. 


\section{DISPERSED TECHNOLOGY CHOICE AND WAITING TIME}

We begin the analysis by assuming that adoption is spread out over some interval $[\tau, T]$. Having derived some results in this way, we shall then show that adoption is indeed spread out as we claim. But first we can rule out a mass of producers adopting the same technology $z$, except possibly at the lowest $z$.

Proposition 1 Suppose $D$ is continuous and that $\phi_{z}^{\prime}>0$ for $z \in[\tau, T]$, and that $N$ is increasing on $[\tau, T]$. Then $N$ is continuous on $[\tau, T]$.

Proof. Let $\xi_{z}=\int_{z}^{\infty} e^{-r(s-z)} p_{s} d s$. Then since each $z \in[\tau, T]$ is chosen by some firm,

$$
V_{z}=e^{-r z}\left(\left[1-\phi_{z}\right] \frac{w}{r}+\phi_{z} \xi_{z}\right)=\frac{w}{r}
$$

is constant on $[\tau, T]$. Therefore $V^{\prime}=V^{\prime \prime}=0$ on $[\tau, T]$. That $V^{\prime \prime}=0$ implies that $\xi^{\prime}$ is continuous. But

$$
\xi^{\prime}=-p_{z}+r \xi
$$

so that $p_{z}=r \xi-\xi^{\prime}$ is continuous. And continuity of $D$ then implies that $N$ too is continuous.

A corollary of proposition 1 is that:

Corollary 1 If $D$ is continuous and that $\phi_{z}^{\prime}>0$ for $z \geq L$, the only possible mass point in $N$ is at $z=L$, and for all $z>L, N$ must be continuous.

Although the conditions referred to in corollary 1 are not the only set of conditions under which technology choices and waiting times would be dispersed in equilibrium, they do refer to an environment most amenable to analysis. We proceed with the analysis under such an environment. First if $N$ has supports over $[\tau, T]$, where $\tau \geq L$, combining (2) and (4) and rearranging, for all $t \in[\tau, T]$,

$$
\frac{w}{r}\left(1+\frac{e^{r t}-1}{\phi_{t}}\right)=\int_{t}^{\infty} e^{-r(s-t)} p_{s} d s
$$


Differentiating with respect to $t$,

$$
\frac{w}{r}\left(\frac{r e^{r t} \phi_{t}-\phi_{t}^{\prime}\left(e^{r t}-1\right)}{\phi_{t}^{2}}\right)=-p_{t}+r \int_{t}^{\infty} e^{-r(s-t)} p_{s} d s .
$$

But from (7),

$$
r \int_{t}^{\infty} e^{-r(s-t)} p_{s} d s=w\left(1+\frac{e^{r t}-1}{\phi_{t}}\right)
$$

and hence

$$
p_{t}=w\left(1+\frac{\phi_{t}^{\prime}}{\phi_{t}^{2}} \frac{e^{r t}-1}{r}-\frac{1}{\phi_{t}}\right) .
$$

This is the solution for $p_{t}, t \in[\tau, T]$.

We must yet determine the interval $[\tau, T]$. At $T$ and thereafter, output becomes stationary, and so does price. If $T$ is the last technology adopted, equilibrium price thereafter is given by

$$
p_{T}=\rho_{T} \equiv w\left(1+\frac{e^{r T}-1}{\phi_{T}}\right) .
$$

But this must be the same as the RHS of (8) when evaluated at $T$. Thus we have the restriction; i.e., the implicit function for $T$ :

$$
\frac{\phi_{T}^{\prime}}{\phi_{T}} \frac{e^{r T}-1}{r}=e^{r T}
$$

Next, we determine $\tau$. There are two cases to consider.

1. $D^{-1}(0)>p_{L}$, where $p_{L}$ is the RHS of (8) evaluated at $t=L$. In order that $p_{\tau}=p_{L}$ when production first begins, there will have to be a mass of entrants $N_{L}$ at $L$ satisfying

$$
p_{L}=D^{-1}\left(N_{L} \phi_{L}\right)
$$

2. $D^{-1}(0)<p_{L}$. Here $\tau$ satisfies

$$
p_{\tau}=D^{-1}(0) \text {, }
$$

in which case $N$ has no mass points throughout. 
All this pins down $p_{t}$ and the interval $[\tau, T]$ on which it is defined. In the above derivation, we assume that there are no holes in $N_{z}$ on $[\tau, T]$. This is guaranteed to be the case as long as long as

1. $p_{t}$, as defined in (8), is strictly decreasing on $[\tau, T] .1$

For $(8)-(12)$ to constitute a unique equilibrium, we also require that

2. $p_{t}$ stays above $w$ on $[\tau, T]$.

3. A unique and positive solution to (10) exists.

4. No one would want to enter outside of the support of $N$; i.e.,

$$
V_{z} \leq \frac{w}{r} \quad \text { for } z \notin[\tau, T]
$$

Proposition 2 A unique equilibrium where technology choices are dispersed, as characterized by (8) - (12), exists if, in addition to the conditions stated in corollary 1,

$$
-\frac{\phi_{t}^{\prime}}{\phi_{t}}\left(e^{r t}-1\right)+r e^{r t}<0
$$

for $t$ arbitrarily close to $L$, and

$$
\frac{\phi_{t}^{\prime \prime}}{\phi_{t}^{\prime}} \leq r
$$

for all $t \geq L$.

We illustrate the idea of the proposition in figure 4. First $\rho_{t}$, which is the long-run equilibrium price as a function of the date when output stabilizes, is plotted as an U-shaped curve. Given that $\phi_{t}$ is bounded below $1, \rho_{t}$, as can be seen from (9), must eventually slope upward. Differentiating (9) yields the LHS of (13). Thus, if (13) holds, $\rho_{t}$ must first slope downward.

\footnotetext{
${ }^{1}$ Suppose there is some subinterval $\left[t_{1}, t_{2}\right]$ within $[\tau, T]$, along which $N$ has no positive support. Then $p_{t}=p_{t_{1}}$, for all $t \in\left[t_{1}, t_{2}\right]$, which contradicts a strictly decreasing $p_{t}$, as given by (8), over the entire $[\tau, T]$ interval.
} 


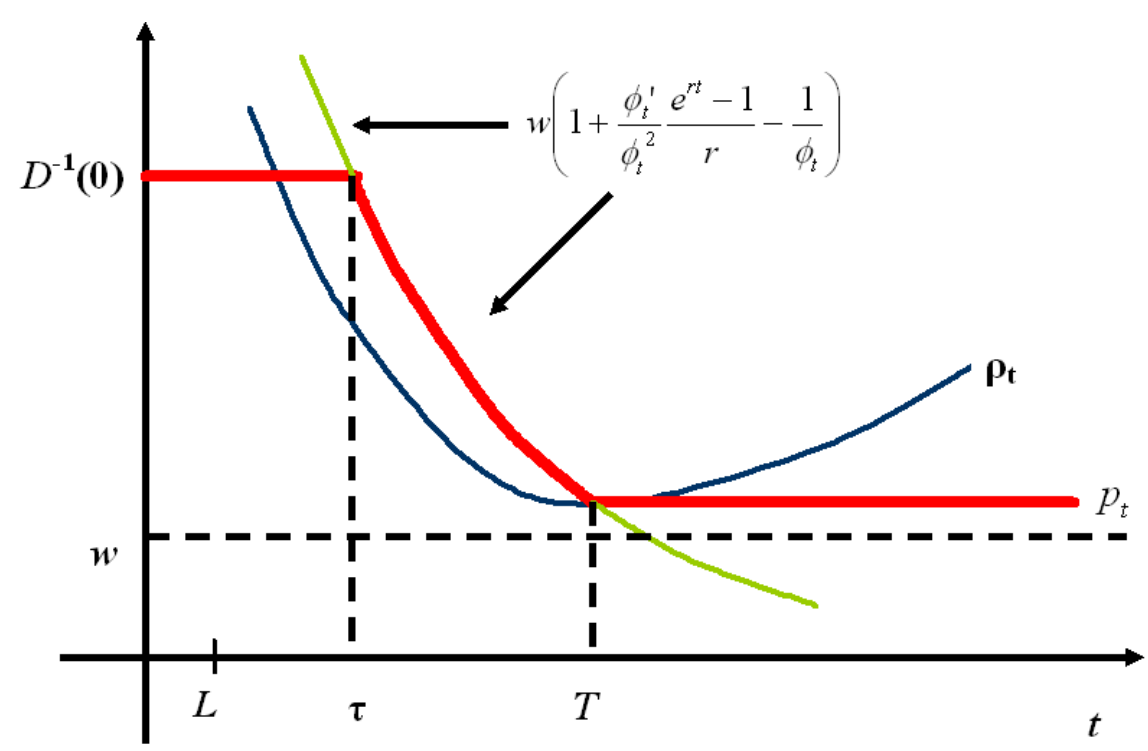

FIG. 4. Existence and uniqueness of equilibrium

Next we can use (13) to verify that, over the interval where $\rho_{t}$ is decreasing, $p_{t}$ as defined by (8) does stay above $w$. Moreover if (14) holds, it is strictly decreasing. ${ }^{2}$ The date when output stabilizes $T$ is given by (10). But then (10) holds at where the LHS of (13) vanishes. Insofar as the LHS of (13) is the slope of $\rho_{t}, T$ is at where $\rho$ is at the minimum. The last technology to be adopted must help bring forth the lowest long-run equilibrium price.

The similarity of price determination in figure 4 with the textbook Marshallian model of a competitive industry is apparent. In the latter model, free entry ensures that, in the long run, the price is equal to the minimum of the U-shaped average cost, at which average and marginal costs are equated. In the present model, the long-

\footnotetext{
${ }^{2}$ Differentiate (8) with respect to $t$ :

$$
\frac{\partial p_{t}}{\partial t}=\left(2\left[r e^{r t}-\frac{\phi_{t}^{\prime}}{\phi_{t}}\left(e^{r t}-1\right)\right]+\left[\left(e^{r t}-1\right)\left(\frac{\phi_{t}^{\prime \prime}}{\phi_{t}^{\prime}}-r\right)\right]\right) \frac{\phi_{t}^{\prime}}{\phi_{t}^{2}} \frac{w}{r},
$$
}

which is guarantied to be negative if (13) and (14) hold. 
run equilibrium price is equal to the minimum of the $\mathrm{U}$-shaped $\rho_{t}$ curve at which the $\rho_{t}$ and the $p_{t}$ curves intersect. This long-run equilibrium is reached gradually as successive cohorts of entrants turn to become bona fide producers over time, in a process akin to how entries are thought to drive individual firms to move down the marginal cost curve in the textbook model.

The final step in establishing the existence of equilibrium is to make sure that no firms find it advantageous to enter outside $[\tau, T]$ : If output stabilized at $T$, for all $t \geq T, p_{t}=\rho_{T}$. Entry at any $z>T$ would have yielded a value equal to $w / r$ if, for all $t \geq z, p_{t}=\rho_{z}$. Now that $T$ minimizes $\rho$, for all $z>T, \rho_{z}>\rho_{T}=p_{T}$. Entering at any $z>T$ must then yield a value below $w / r$.

In figure 4 , we assume $D^{-1}(0)<p_{L}$, so that $p_{\tau}=D^{-1}(0)$. In this case, the value of entering at some $z<\tau$ would have been equal to $w / r$ if $p_{t}$ had followed (8) for all $t \in[z, T]$. But for all $t \in[0, \tau], p_{t}=D^{-1}(0)$ instead. Entering at any $z<\tau$ must then yield a value below $w / r$. In case $D^{-1}(0)>p_{L}, \tau=L$. Clearly, no one would want to enter at any $z<L$, with $\phi_{z}$ assumed equal to 0 for all such waiting times.

The key to dispersed technology choice is condition (13), which can be rewritten as

$$
\frac{\phi_{t}^{\prime}}{\phi_{t}}>\frac{r e^{r t}}{e^{r t}-1} .
$$

This is the counterpart of (6) in the 2-technology example. In the present analysis and in the 2-technology example, whether or not firms would ever wish to wait out longer than is absolutely necessary before attempting production hinges on whether there would be a lower long-run equilibrium price to follow. In turn, this will be the case when the profile of success probability is sufficiently steep over a range of technology choice. That is, when (15) above and (6) for the 2-technology example hold.

We now resume to complete the characterization of equilibrium. When the conditions of proposition 2 are met, $N$ is differentiable. This allows us to determine $n_{t}$ as 
follows: From (3),

$$
\int_{\tau}^{t} \phi_{s} n_{s} d s=D\left(p_{t}\right)
$$

Differentiating and rearranging,

$$
n_{t}=\frac{1}{\phi_{t}} D^{\prime}\left(p_{t}\right) \frac{d p_{t}}{d t} .
$$

Since we know $p_{t}$ we also know $d p_{t} / d t$, and so we have $n_{t}$.

\section{LEARNING CURVE AND INDUSTRY LIFECYCLE - TWO EXAMPLES}

Our goal in this section is to describe how a new industry would evolve under two different scenarios : $(i)$ when very little is known about what would work in the new industry, and $(i i)$ when the technology used in the new industry is expected to be very similar to those used elsewhere. In the first scenario, the payoffs for waiting out longer before attempting production to learn what may work could be bountiful. This corresponds to, in our model, a $\phi_{z}$ function that rises rather gradually as $z$ increases. Learning in the industry is primarily active in the sense of Ericson and Pakes $(1995,1998)$, where an individual firm's survival is largely determined by its choice of learning investment in terms of time lost to production. In the second scenario, on the contrary, the necessary adaptations of existing technologies would be relatively minor. The $\phi_{z}$ function in such an industry would rise toward its upper bound relatively rapidly. Learning in this case is primarily passive in the sense of Jovanovic (1982), where learning investment plays a relative minor role in an individual firm's survival probability, which depends largely on the firm's initial endowement.

Specifically, we examine the technology choices and evolutions of $p_{t}$ and $q_{t}$ under two specifications of $\phi_{z}$ :

1. $\phi_{z}^{S}=0.01 z^{2}-0.00038 z^{3}$, 
2. $\phi_{z}^{R}=0.105 z+0.018 z^{2}-0.002 z^{3}$

for $z \geq L=0.5$. The two learning curves are those plotted in figure 1 . In the analysis to follow, we set $r=0.05$ to give a unit of time an interpretation of a year, and assume $w=1$ and a demand curve: $D(p) \equiv(12-p)^{6}$, whereby $D^{-1}(0)=12$.

The major implication of our analysis is that diffusion should be more gradual when there is a longer range of waiting times over which $\rho_{z}$ remains downward sloping, or equivalently, when there is a longer range of waiting times over which $\phi_{z}$ remains relatively steep. Hence, the major difference in the life-cycles of the two industries seems to be that there should be more gradual and protracted output increase under the more gentle learning curve $\phi_{z}^{S}$ (active learning) than under the more rapid learning curve $\phi_{z}^{R}$ (passive learning).

\begin{tabular}{|c|c|c|c|c|}
\hline & $\tau$ & $T$ & $T-\tau$ & $p_{T} / p_{\tau}$ \\
\hline$\phi_{z}^{S}$ & 3.25 & 10.87 & 7.62 & 0.17 \\
$\phi_{z}^{R}$ & 0.5 & 3.57 & 3.07 & 0.69 \\
\hline
\end{tabular}

In table 1, we report the various characteristics of the life-cycles of the two industries. Under $\phi_{z}^{S}, p_{L}>D^{-1}(0)$, and there are no mass points throughout $N$. In this case, $\tau$ is pinned down by $p_{\tau}=D^{-1}(0)=12$. Under $\phi_{z}^{R}, p_{L}<D^{-1}(0)$, however, and so $\tau=L$, and equilibrium is supported by a mass of entrants at $\tau=L$. Under the more gentle learning curve $\phi_{z}^{S}$, where learning is primarily active, there is indeed more protracted diffusion inasmuch as $[\tau, T]$ spans a longer time interval than when learning is relatively rapid and passive as under $\phi_{z}^{R}$. To accompany the more protracted diffusion, industry $\phi_{z}^{S}$ also experiences a more substantial fall in $p_{t}$, and therefore a greater output increase over time. That output increase should be more gradual but also more substantial under active, as opposed to passive, learning is a conclusion also reached in Ericson and Pakes (1998) under rather different specifications of the two 
types of learning. Perhaps then this difference should be considered as a rather robust prediction of how, in a broad sense, active learning compares with passive learning.

Output increase under $\phi_{z}^{S}$ is not just more protracted but also more $S$-shaped than under $\phi_{z}^{R}$, as shown in figures 5 and 6 , respectively. The $S$-shaped diffusion curve, along which output first increases at an increasing rate, has been reported to well describe the pattern of output increase in many industries since Griliches (1957). Figures 7 and 8 show, respectively, that technology choices under $\phi_{z}^{S}$ are more dispersed and bell-shaped than under $\phi_{z}^{R}{ }^{3}$ The $S$-shaped diffusion curve in figure 5 then seems to have been a result of the bell-shaped pattern of technology choice in figure 7 : Output would increase at an increasing rate for a period of time if $n_{t}$ increases rapidly in the interim. Previous theoretical analyses largely rely on some kinds of ex-ante heterogeneity among firms or external effect, whether explicit or implicit, to help generate a $S$-shaped diffusion curve. ${ }^{4}$ In contrast, the present analysis shows that a $S$-shaped diffusion curve may arise in the entire absence of any ex-ante heterogeneity among producers and non-pecuniary externality.

Finally, the present analysis suggests that passive and active learning may also be distinguished by the patterns of exit. If under passive learning, the choices of lead times tend to cluster temporally as shown in figure 8, so do exits. Then the shakeout, defined by Gort and Klepper (1982) as the episode when the number of producers in an industry is falling from its peak, comes sooner, is more discernable, and ends more abruptly. ${ }^{5}$

\footnotetext{
${ }^{3}$ In fact, technology choices under $\phi_{z}^{R}$ are even more concentrated at the first available technology $z=L$ than is shown in figure 8, where a mass of entrants adopting $z=L$ is not depicted in the figure.

${ }^{4}$ Jovanovic and Lach (1989) and Davies (1979), for example. See also the surveys in Lissoni and Metcalfe (1994) and Geroski (2000).

${ }^{5}$ Jovanovic and Tse (2006), in a vintage capital model, find that the shakeout should come sooner when technological change proceeds at a faster pace. The present analysis presumes a shakeout a
} 


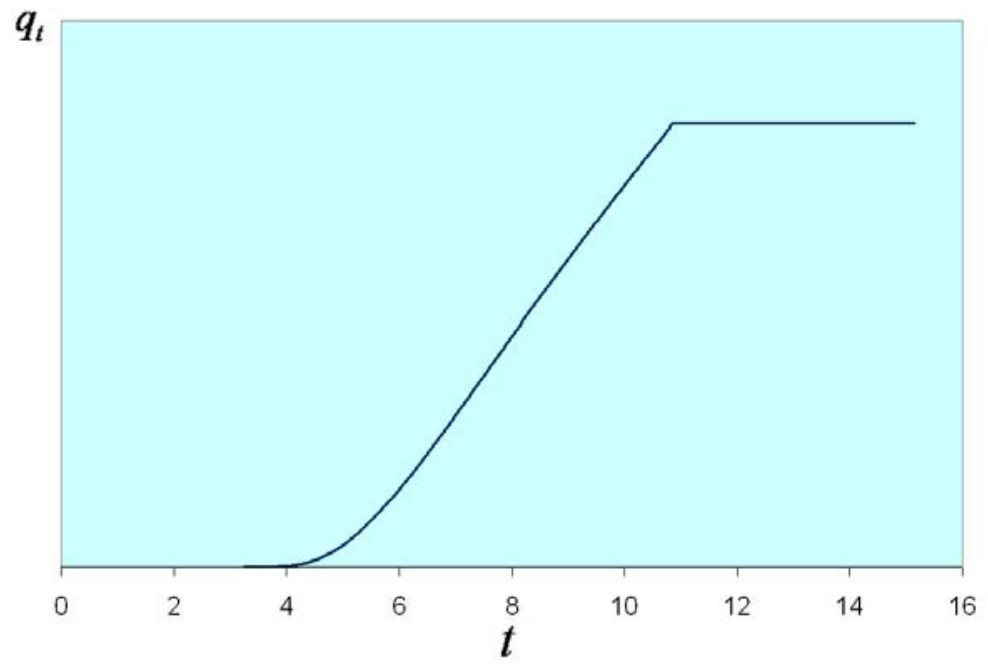

FIG. 5. $q_{t}$ under $\phi_{z}^{S}$ : A more protracted and S-shaped diffusion

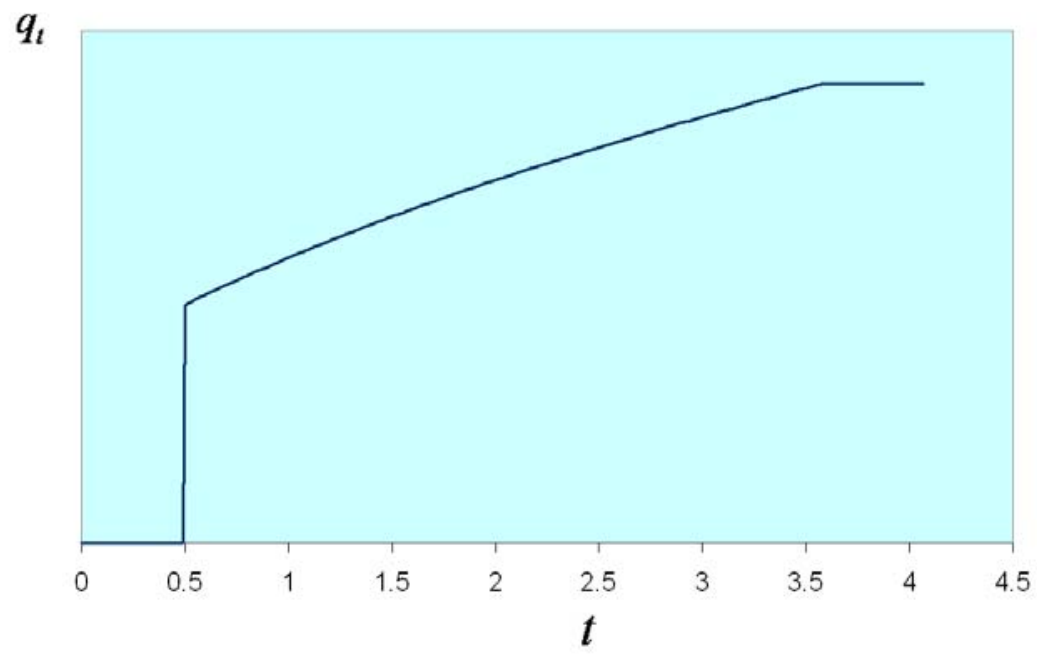

Fig. 6. $q_{t}$ under $\phi_{z}^{R}$ : A more rapid diffusion 


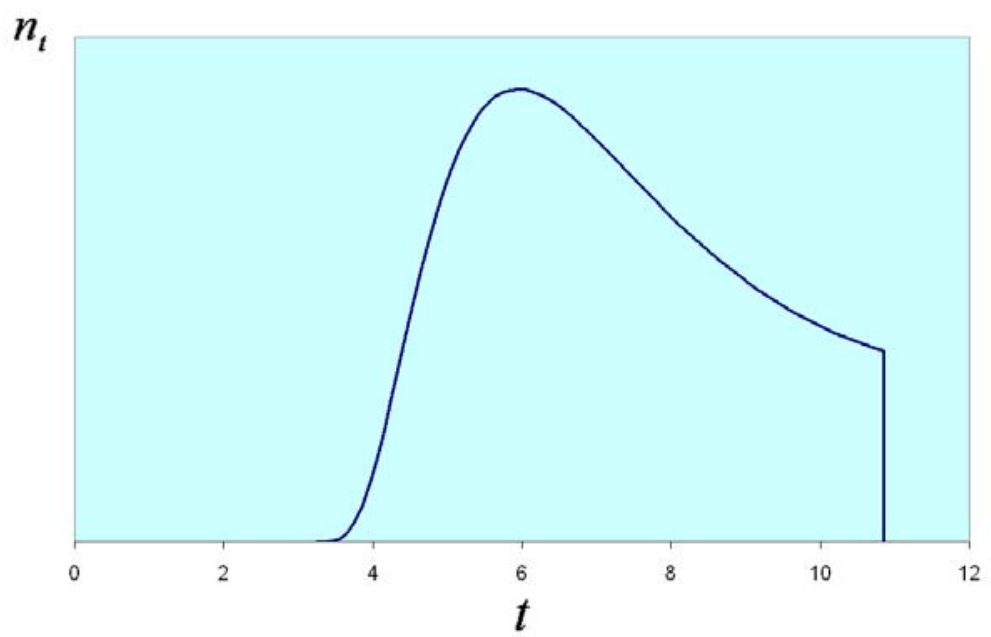

FIG. 7. $n_{t}$ under $\phi_{z}^{S}$ : Bell-shaped and dispersed

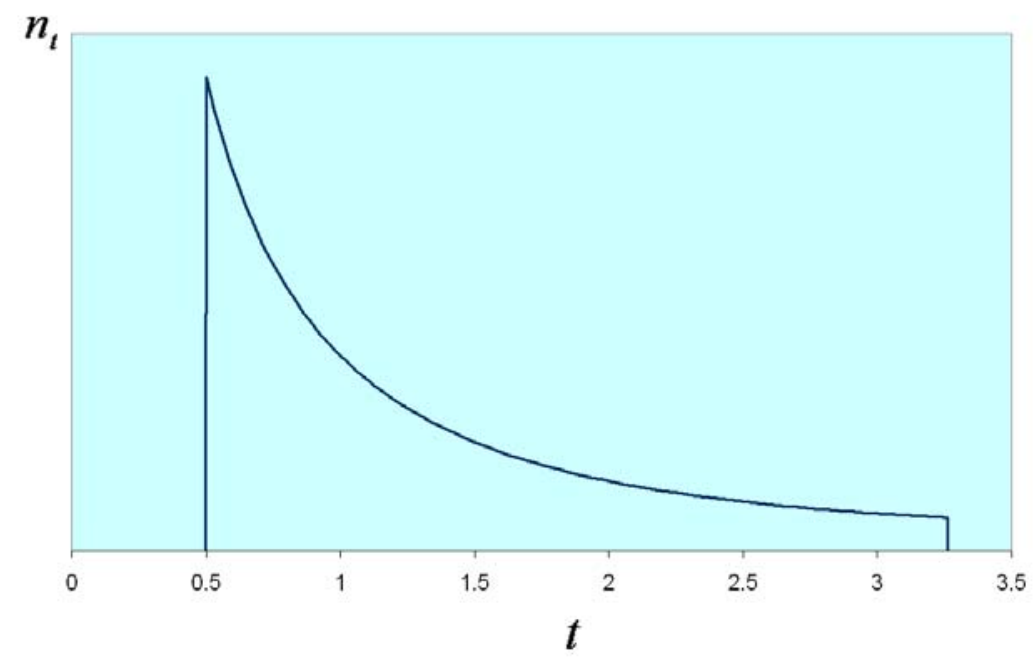

FIG. 8. $n_{t}$ under $\phi_{t}^{R}$ :Skewed and concentrated 


\section{MARKET SIZE, INCOME DISTRIBUTION, AND DIFFUSION}

A ubiquitous implication of models of learning by doing is that market size and the distribution of income among consumers, through their impacts on demand, could play important roles in determining the pace of diffusion (Jovanovic and Lach (1989); Matsuyama (2002)) A larger population of all consumers in general and a larger population of high income consumers in particular raise the profitability of entry and investment at the outset. Subsequent entrants then benefit from the industry-wide learning by doing that originated from the higher initial entry. All this speeds up the diffusion to low income consumers. The present model is a model of learning by doing in which the time that an entrant must wait before production commences is a period of learning and adaptation. But where the benefits of learning are purely internal, demand plays no role in determining the pace of diffusion.

The argument can be formalized as follow. Suppose the market is populated by a continuum of $H$ consumers, each of whom share the same preference :

$$
u=\mathcal{I} v+x,
$$

where $\mathcal{I}=1$ if the consumer buys a unit of the good and 0 otherwise, $v$ the value the consumer attributes to the consumption of the good, and $x$ the quantity of an outside good consumed. Consumer $i$ has income $y_{i}$ to be allocated between the purchases of the two goods:

$$
\mathcal{I} p+x \leq y_{i}
$$

where we normalize the price of the outside good to 1 . No borrowing nor lending is allowed. Hence if $y_{i}<p$, consumer $i$ spends his entire budget on the outside good. priori, and is concerned with factors that determine the characters of the shakeout. This stands in contrast but is complementary to Utterback and Suarez (1993), Hopenhayn (1993), Jovanovic and MacDonald (1994), Klepper and Miller (1995) and Klepper (1996, 2002) and Jovanovic and Tse (2006), whose focuses are on factors that help give rise to the shakeout. 
If $y_{i} \geq p$, the good becomes affordable, and consumer $i$ will buy one unit if $v>p$ as well. That is, consumer $i$ will buy if $p \leq \min \left\{y_{i}, v\right\}$. Let the distribution of income among consumers be given by $G(y)$, with upper support equal to $\bar{y}$. If $v>\bar{y}$, the quantity demanded at price $p$ is given by

$$
q=H(1-G(p))
$$

As $p$ falls over time, the good will reach more and more consumers. Specifically, the good will diffuse to consumer $i$ at time $t$, where $t$ solves $p_{t}=y_{i}$. In the present model, however, $p_{t}$ is entirely determined by $\phi_{z}$ and $w$; it is invariant to the parameters of the demand curve. Neither market size $H$ nor the distribution of income $G$ has any effects at all on when a consumer with a given income may first start to buy.

Market size and income distribution affect equilibrium only in terms of $q_{t}$ and $n_{t}$. By (17), with $p_{t}$ staying at the same level as $H$ varies, $q_{t}$ will rise in straight proportion to increases in $H$. The same conclusion applies to $n_{t}$ too, as can be seen by a simple substitution of (17) into (16).

\section{MONOPOLY}

In competitive markets, we find that the long-run equilibrium price minimizes $\rho_{z}$. The extent of dispersion in technology choice and the rapidity of diffusion depends on for how far $\rho_{z}$ remains decreasing. Does a monopoly tend to adopt a wider or a narrower range of waiting times? Or equivalently, does market power result in more or less rapid diffusion?

Consider a monopoly hiring some $M_{T}$ managers at date 0 , each of whom will be assigned to work on a given technology. When the waiting time of a given technology is over, the monopoly will release the assigned manager if the technology fails to deliver. The wage per unit of time is the opportunity cost of managers, $w$. The monopoly chooses the number of managers to be assigned to technology $z=t, m_{t}$, 
to maximize

$$
\Pi=\int_{0}^{\infty} e^{-r t}\left[D^{-1}\left(q_{t}\right) q_{t}-\left(M_{T}-X_{t}\right) w\right] d t
$$

subject to

$$
\begin{gathered}
\frac{d q_{t}}{d t}=m_{t} \phi_{t}, \quad q_{0}=0 ; \\
\frac{d X_{t}}{d t}=m_{t}\left(1-\phi_{t}\right), \quad X_{0}=0 ; \\
M_{t}=\int_{0}^{t} m_{s} d s .
\end{gathered}
$$

In (18) and (20), $X_{t}$ denotes cumulative exit at time $t$, and hence $M_{T}-X_{t}$ denotes the number of managers who remain employed by the monopoly at time $t$. We prove in the appendix that:

Proposition 3 Assuming the conditions in proportion 2 are met, the monopoly chooses the same interval $[\tau, T]$ on which $m_{z}>0$ as the interval selected in competition. For each $t \in[\tau, T]$, the monopoly's optimum satisfies

$$
\mu_{t}=w\left(1+\frac{\phi_{t}^{\prime}}{\phi_{t}^{2}} \frac{e^{r t}-1}{r}-\frac{1}{\phi_{t}}\right),
$$

where

$$
\mu_{t}=\left(D^{-1}\right)^{\prime}\left(q_{t}\right) q_{t}+p_{t}
$$

is the monopoly's marginal revenue.

The next step is to determine whether there would be any systematic bias for the monopoly to cluster his technology choices around any particular $z \in[\tau, T]$ relative to the profile of technologies chosen in competition. The answer seems to be no. Observe that the competitive equilibrium price coincides with the left side of (22). True, the monopoly produces less at each $t$, but otherwise, its marginal revenue is set equal to the competitive price at each $t$. Hence the output restriction at each $t$ is, roughly 
speaking, uniform. If we assume a demand curve of the form $q_{t}=A\left(\bar{p}-p_{t}\right)^{\alpha}$, for some $A>0, \alpha>0$, and $\bar{p} \geq 0$, we can show that

$$
m_{z}=n_{z}\left(\frac{\alpha}{1+\alpha}\right)^{\alpha} .
$$

Where $m_{z}$ is proportional to $n_{z}$, the probability densities of technology choice under monopoly and competition just coincide for all $z \in[\tau, T]$.

\section{CONCLUSION}

This paper started out arguing that entrants to a new industry could face nontrivial choices among technologies with different lead times and success probabilities. We then showed that how in this environment, there can be gradual diffusion, and how competition minimizes the long-run equilibrium price, via selecting the technology consistent with the least long-run price as the last technology to be adopted. When learning opportunities are more bountiful, diffusion tends to be more gradual and exits more spread out. On the contrary, when learning opportunities are more limited, the choices of lead times and thereby exits would cluster temporally. Furthermore, we showed how under internal, as opposed to external, learning by doing, demand plays at most a minimal role in determining the rate of diffusion. Finally, a similar conclusion holds with respect to market structure, whereby a monopoly would choose a rate of diffusion similar to the rate of diffusion under competition.

\section{APPENDIX}

\section{Proof of proposition 3.-}

The problem is almost a standard optimal control problem, except for the integral constraint in (21). To make it amendable to standard optimal control techniques, we define a new state variable:

$$
\gamma_{t}=-\int_{0}^{t} m_{s} d s
$$


whereby

$$
\frac{d \gamma_{t}}{d t}=-m_{t}
$$

The initial and terminal conditions on $\gamma_{t}$ are, respectively,

$$
\gamma_{0}=0 \quad \text { and } \quad \gamma_{T}=-M_{T}
$$

where the latter is simply a restatement of (21). The integral constraint in (21) can thus be replaced by (24) and (25). Further, rewrite (18) as

$$
\Pi=\Pi^{1}-\frac{w}{r} M_{T},
$$

where

$$
\Pi^{1}=\int_{0}^{\infty} e^{-r t}\left[D^{-1}\left(q_{t}\right) q_{t}+X_{t} w\right] d t .
$$

The maximization of $\Pi$ proceeds in two stages. First, we fix $M_{T}$ at some arbitrary value and maximize $\Pi^{1}$ subject to (19), (20), (24), and (25). This step yields the maximized value of $\Pi^{1}$ as a function of $M_{T}$. We then proceed to maximize $\Pi$ with respect to $M_{T}$.

Step 1: Write the Hamiltonian of (27) as

$$
\mathcal{H}_{t}=e^{-r t}\left[D^{-1}\left(q_{t}\right) q_{t}+X_{t} w\right]+\lambda_{t}^{q} m_{t} \phi_{t}+\lambda_{t}^{X} m_{t}\left(1-\phi_{t}\right)-\lambda_{t}^{\gamma} m_{t}
$$

where $\lambda_{t}^{q}, \lambda_{t}^{X}$, and $\lambda_{t}^{\gamma}$ are the co-state variables of the respective constraints. The necessary conditions for maximum are

$$
\begin{gathered}
\left.\lambda_{t}^{q} \phi_{t}+\lambda_{t}^{X}\left(1-\phi_{t}\right)-\lambda_{t}^{\gamma} \leq 0 \quad \text { (with equality if } m_{t}>0\right), \\
\frac{d \lambda_{t}^{q}}{d t}=-e^{r t} \mu\left(q_{t}\right), \quad \text { where } \mu\left(q_{t}\right)=\left(D^{-1}\right)^{\prime}\left(q_{t}\right) q_{t}+D^{-1}\left(q_{t}\right), \\
\frac{d \lambda_{t}^{X}}{d t}=-e^{-r t} w, \\
\frac{d \lambda_{t}^{\gamma}}{d t}=0,
\end{gathered}
$$




$$
\lim _{t \rightarrow \infty} \lambda_{t}^{q}=\lim _{t \rightarrow \infty} \lambda_{t}^{X}=0,
$$

in addition to (19), (20), (24), and (25). Now by (31), $\lambda_{t}^{\gamma}$ is time-stationary, and we may drop the time subscript. Integrating both sides of (30), while making use of (32), yields

$$
\lambda_{t}^{X}=e^{-r t} \frac{w}{r} .
$$

Suppose $m_{t}>0$ over some interval $[\tau, T]$. For $t \in[\tau, T],(28)$ holds as an equality:

$$
\lambda_{t}^{q}=\frac{1}{\phi_{t}}\left(\lambda^{\gamma}-e^{-r t} \frac{w}{r}\left(1-\phi_{t}\right)\right),
$$

where we have made use of (33) to substitute out $\lambda_{t}^{X}$. Differentiating:

$$
\frac{d \lambda_{t}^{q}}{d t}=-\frac{\phi_{t}^{\prime}}{\phi_{t}^{2}}\left(\lambda^{\gamma}-e^{-r t} \frac{w}{r}\left[1-\phi_{t}\right]\right)+\frac{1}{\phi_{t}}\left(e^{-r t} w\left[1-\phi_{t}\right]+e^{-r t} \frac{w}{r} \phi_{t}^{\prime}\right) .
$$

Setting the RHS of the above equal to the RHS of (29) and simplifying,

$$
\mu\left(q_{t}\right)=\frac{\phi_{t}^{\prime}}{\phi_{t}^{2}} e^{r t} \lambda^{\gamma}-\frac{w}{r}\left[\frac{\phi_{t}^{\prime}}{\phi_{t}^{2}}+\frac{1-\phi_{t}}{\phi_{t}} r\right]
$$

This characterizes the time-path of output over the interval $[\tau, T]$ as a function of $\lambda^{\gamma}$.

Step 2: $\lambda^{\gamma}$, as the Lagrange multiplier of $(24)$ and (25) in the maximization of $\Pi^{1}$, satisfies ${ }^{6}$

$$
\frac{\partial \Pi^{1}}{\partial \gamma_{T}}=-\lambda^{\gamma}
$$

But $\gamma_{T}=-M_{T}$; hence

$$
\frac{\partial \Pi^{1}}{\partial M_{T}}=\lambda^{\gamma}
$$

The first order condition of maximizing (26) is then

$$
\lambda^{\gamma}=\frac{w}{r}
$$

Substituting the above into (34) and simplifying yield (22). The boundaries of the interval $[\tau, T]$ are yet to be determined. When $m_{t}$ first turns positive, $q_{t}=0$. At zero

\footnotetext{
${ }^{6}$ See Chiang (1992), p.206.
} 
output, the marginal revenue of the monopoly is simply $D^{-1}(0)$. Then $\tau$ solves

$$
D^{-1}(0)=\left(1+\frac{\phi_{\tau}^{\prime}}{\phi_{\tau}^{2}} \frac{e^{r \tau}-1}{r}-\frac{1}{\phi_{\tau}}\right) w .
$$

This derivation assumes $D^{-1}(0)$ exceeds the RHS of the above evaluated at $\tau=$ $L$. Were this condition not met, $\tau=L$, analogous to how $\tau$ is pinned down in competitive markets in such circumstances. To determine $T$, observe that the value to the monopoly of hiring the last manager is

$$
\phi_{T} \int_{T}^{\infty} \mu\left(q_{T}\right) e^{-r t} d t
$$

whereas the cost is

$$
\int_{0}^{T} w e^{-r t} d t+\phi_{T} \int_{T}^{\infty} w e^{-r t} d t
$$

and they must be equalized to maximize profit. This yields, after some simplification,

$$
\mu\left(q_{T}\right)=\frac{1-e^{-r T}\left(1-\phi_{T}\right)}{e^{-r T} \phi_{T}} w .
$$

Equating the RHS of this equation to the RHS of (22) and evaluating at $T$ yields exactly (10).

\section{REFERENCES}

[1] Alchian, A. (1959): "Costs and Outputs," in M. Abramovitz et al. (eds), The Allocation of Economic Rescources, Stanford University Press.

[2] Bahk, B.-H. and Gort, M. (1993): "Decomposing Learning by Doing in New Plants," Journal of Political Economy 101: 561-583.

[3] Basu, S. and Weil, D. N. (1988): "Appropriate Technology and Growth," Quarterly Journal of Economics 113: 1025-1054.

[4] Chiang, A. (1992): Elements of Dynamic Optimization, McGraw-Hill Inc. 
[5] Davies, S. (1979): The Diffusion of Process Innovations, Cambridge University Press, Cambridge.

[6] Geroski, P. A. (2000): "Models of technology diffusion," Research Policy 29: 603-625.

[7] Girliches, Z. (1957): "Hybrid Corn: An Exploration in the Economics of Technological Change," Econometrica 25: 501-522.

[8] Gort, M. and Klepper, S. (1982): "Time Paths in the Diffusion of Market Innovations," Economic Journal 92: 630-653.

[9] Hopenhayn, H. (1993): "The Shakeout," Universitat Pompeu Fabra working paper 33.

[10] Jovanovic, B. (1982): "The Selection and Evolution of Industry," Econometrica 50: 649-670.

[11] — (2004): "The Pre-producers," NBER working paper 10771.

[12] — and Lach, S. (1989): "Entry, Exit, and Diffusion with Learning by Doing," American Economic Review 79: 690-699.

[13] — and MacDonald, G. (1994): "The Lifecycle of a Competitive Industry," Journal of Political Economy 102: 322-347.

[14] — and Tse, C.-Y. (2006): "Creative Destruction in Industries," NBER working paper 12520.

[15] Klepper, S. (1996): "Entry, Exit, and Innovation over the Product Life Cycle," American Economic Review 86: 562-583.

[16] — (2002): "Firm survival and the evolution of oligopoly," Rand Journal of Economics 33: $37-61$. 
[17] — and Miller, J. H. (1995): "Entry, exit, and shakeouts in the United States in new manufactured products," International Journal of Industrial Organization 13: $567-591$.

[18] Lissoni, F. and Metcalfe, J.S. (1994): "Diffusion of Innovation Acient and Modern: A Review of the Main Themes," in M. Dodgson and R. Rothwell (eds), The Handbook of Industrial Innovation, Edward Elgar.

[19] Matsuyama, K. (2002): "The Rise of Mass Consumption Societies" Journal of Political Economy 110: 1035-1070.

[20] Pakes, A. and Ericson, R. (1995): "Markov-Perfect Industry Dynamics: A Framework for Empirical Work," Review of Economic Studies 62: 53-82.

[21] — (1998): "Empirical Implications of Alternative Models of Firm Dynamics," Journal of Economic Theory 79: 1-45.

[22] Reinganum, J. (1981): "Market Structure and the Diffusion of New Technology," Rand Journal of Economics 12: 618-624.

[23] Utterback, J. and Suarez, F. (1993): "Innovation, Competition, and Industry Structure," Research Policy 22: 1-21. 